\title{
EIN LINEARES OPTIMIERUNGSMODELL ZUR PRODUKTIONSPLANUNG BEI ZYKLISCHER KUPPELPRODUKTION
}

\author{
Christoph Pesch, Henkel KGaA, Düsseldorf
}

In der Fettchemie werden native öle und Fette verarbeitet und die daraus erzeugten Produkte vermarktet. Die größten Produktgruppen sind Fettsäuren, Methylester und Fettalkohole.

Bei dem eingesetzten DV-System handelt es sich um ein MehrperiodenPlanungsmodell. Es wird zur Monats- bzw. Jahresplanung und für Alternativrechnungen (Rohstoffe, Verfahren, Verkaufsprogramme) genutzt. Es können sowohl einzelne, als auch mehrere Produktionsstandorte des Unternehmens betrachtet werden. ziel ist es, den optimalen Einsatz von stoffen und Kapazitäten zur Erfüllung des Verkaufsprogramms $z$ u berechnen, so daß entweder die Gesamtkosten minimiert oder der Gewinn maximiert wird. Vorhandene Absatz-, Beschaffungs- und Kapazitätsrestriktionen werden berücksichtigt.

Die Vielzahl der visschiedenen Verfahrenswege, die Verwendung der Kuppelprodukte bei zykiischer Kuppelproduktion und der Eirısatz alternativer Rohstoffe machen die Möglichkeiten der Auswahl und zuordnungen so groß, daß eine akzeptable Lösung wegen der differenzierten Produkte auf allen Ebenen und der Kapazitätsbeschränkungen der Anlagen nur durch ein lineares optimierungsmodell gefunden werden kann. Dazu wird die optimierungssoftware OSL (Optimization Subroutine Library) der IBM auf einer RS6000 eingesetzt.

Alle dafür benötigten Daten werden in einer Datenbank verwaltet und gepflegt. Die Datenerfassung und die Auswertungen der optimierungsergebnisse sind so organisiert, daß der Benutzer nur mit ihm bekannten Schlüsseln wie Artikelnummer, stoffnummer, Rezepturnummer usw., arbeitet und die speziellen Dateien, die die optimierungssoftware benötigt, weder sieht noch bearbeitet.

Das heutige Angebot von Hard- und Software erlaubt es, Modelle mit 14.000 zeilen und 38.000 Spalten in vertretbarer Zeit zu lösen. 